\title{
1 Scaleable production of microbubbles using an ultrasound-modulated microfluidic device
}

2 Dario Carugo, ${ }^{1 \uparrow}$ Richard J. Browning, ${ }^{2, \pi}$ Ida Iranmanesh, ${ }^{2}$ Walid Messaoudi, ${ }^{3}$ Paul Rademeyer, ${ }^{2}$ and

3 Eleanor Stride $2 \mathrm{a}$

$4{ }^{1}$ Department of Pharmaceutics, UCL School of Pharmacy, University College London (UCL), UK

$5{ }^{2}$ Institute of Biomedical Engineering, Department of Engineering Science, University of Oxford, UK

$6 \quad{ }^{3}$ Faculty of Engineering and Physical Sciences, University of Southampton, UK

7 "Authors have equally contributed to the work in this study

8 Surfactant-coated gas microbubbles are widely used as contrast agents in ultrasound imaging and

9 increasingly in therapeutic applications. The response of microbubbles to ultrasound can be strongly

10 influenced by their size and coating properties and hence the production method. Ultrasonic

11 emulsification (sonication) is the most commonly employed method and can generate high

12 concentrations of microbubbles rapidly, but with a broad size distribution and there is a risk of

13 contamination and/or degradation of sensitive components. Microfluidic devices provide excellent

14 control over microbubble size, but are often challenging or costly to manufacture, offer low

15 production rates $\left(<10^{6} \mathrm{~s}^{-1}\right)$, and are prone to clogging. In this study, a hybrid sonication-microfluidic

16 or 'sonofluidic' device was developed. Bubbles of $\sim 180 \mu \mathrm{m}$ diameter were produced rapidly in a T-

17 junction and subsequently exposed to ultrasound $(71-73 \mathrm{kHz})$ within a microchannel, generating

18 microbubbles (mean diameter: $1-2 \mu \mathrm{m}$ ) at a rate of $>10^{8} \mathrm{~s}^{-1}$ using a single device. Microbubbles were

19 prepared using either the sonofluidic device or conventional sonication and their size, concentration

20 and stability compared. The mean diameter, concentration and stability were found to be

21 comparable between techniques, but the microbubbles produced by the sonofluidic device were all

$22<5 \mu \mathrm{m}$ in diameter and thus did not require any post-production fractionation.

a eleanor.stride@,eng.ox.ac.uk 


\section{I. INTRODUCTION}

\section{A. Microbubbles in ultrasound imaging and therapy}

In medical imaging, microbubbles are routinely used as ultrasound contrast agents. Their high

26 compressibility enables significant enhancement of ultrasound backscatter from blood by several

27 orders of magnitude. The microbubble core usually consists of a high molecular weight gas (e.g. a

28 perfluorocarbon or sulfur hexafluoride) stabilised by a surfactant or polymer coating (or "shell") to

29 enhance stability during storage, handling, and/or administration (Stride and Saffari 2003). Typical

30 clinical formulations use saturated phospholipids or denatured albumin as the primary shell

31 constituents. Moreover, microbubbles can be loaded with biologically active compounds or

32 functionalised with targeting moieties. This has paved the way for their use as targetable drug delivery

33 systems, whereby the bioactive payload can be released on-demand upon extracorporeal ultrasound

34 stimulation directed to the point of treatment (Ferrara, et al. 2007, Kooiman, et al. 2014).

35 The clinical utility of microbubbles is profoundly influenced by their physical characteristics,

36 including their average size, size distribution, and the mechanical and rheological properties of the

37 coating layer (Alter, et al. 2009, Garg, et al. 2013, Sirsi, et al. 2010). These characteristics are in turn

38 dependent on the chemical formulation of the microbubble shell and also on the production technique

39 (Al-Jawadi and Thakur 2020, Hosny, et al. 2013). A variety of different methods have been developed

40 for batch production of microbubbles, including ultrasonic emulsification (sonication), high shear

41 emulsification, membrane emulsification, and coaxial electrohydrodynamic atomisation (Stride and

42 Edirisinghe 2008). Sonication is the most commonly employed method in both academic and

43 industrial laboratories, and involves dispersing gas or liquid in a suspension of a coating material using

44 high intensity ultrasound (Stride, et al. 2020). The size distribution of microbubbles obtained from

45 sonication is however relatively broad (Feshitan, et al. 2009) and there is also wide variability in coating

46 properties (Browning, et al. 2019), which may lead to a large variation in acoustic response across a 
47 microbubble population (Rademeyer, et al. 2015). Post-production procedures (i.e., fractionation or

48 filtration) are usually required in order to remove large bubbles (i.e., with diameters $>10 \mu \mathrm{m}$ ) that

49 could cause vascular occlusion after intravenous injection, as well as excess coating material not

50 incorporated onto the microbubble (Dewitte, et al. 2019, Feshitan, et al. 2009). The latter is particularly

51 important in the case of drug-loaded microbubbles, to enable accurate quantification of the

52 administered dose.

53 B. Microfluidic techniques for microbubble production

54 More recently, microfluidic techniques have been proposed as an alternative to batch methods

55 for producing more uniform microbubbles (Chen, et al. 2014, Dhanaliwala, et al. 2013, Gnyawali, et

56 al. 2017, Hettiarachchi, et al. 2007, Jiang, et al. 2016, Peyman, et al. 2012, Rickel, et al. 2018, Segers, et

57 al. 2020, Seo, et al. 2010, van Elburg, et al. 2021). A typical microfluidic device consists of a cross-flow

58 (i.e., flow focusing) or T-junction architecture, in which gas and liquid streams are forced to flow into

59 a confined microchannel where the gas stream breaks up into individual microbubbles, a process often

60 referred to as 'pinch-off' (Garstecki, et al. 2006, Pahlavan, et al. 2019). Microbubbles produced with

61 this technique typically have a polydispersity index $<5 \%$. However, production of microbubbles

62 having clinically applicable diameters (i.e., in the range 1-10 $\mu \mathrm{m}$ ) requires microchannel features of 63 comparable dimensions (Hettiarachchi, et al. 2007), which can reduce a device's lifetime considerably

64 (i.e., due to clogging or excessive backpressure). Depending on the geometrical properties of the 65 microchannels and the flow dynamic field, different microbubble production regimes have been 66 demonstrated using these architectures (Dollet, et al. 2008). However, production rates are typically

67 lower compared with batch methods (Table 1), and microbubble stability has also been reported to be

68 lower in some cases (Hosny, et al. 2013). These factors have hindered the adoption of microfluidics

69 for industrial production of microbubbles for clinical usage. 
Some of these limitations can be addressed through changes to the operating conditions

71 and/or the device architecture. For example, Peyman et al. were able to achieve a micro-spraying

72 microbubble formation regime by varying the geometry of a flow-focusing microfluidic device,

73 specifically by widening the exit channel and introducing an abrupt increase in the channel depth.

74 When compared to a more conventional pinch-off formation regime, micro-spraying resulted in 75 microbubble suspensions having $\sim 100$-times greater concentration (i.e., up to $10^{9}$ microbubbles $/ \mathrm{mL}$ )

76 (Peyman, et al. 2012). However, the size distribution of microbubbles produced by micro-spraying

77 presented comparable relative standard deviation to that of microbubbles produced by batch 78 mechanical agitation; although the latter method generated some microbubbles with diameter $>10 \mu \mathrm{m}$

79 that were not present in the microfluidic-generated samples. Castro-Hernández et al. demonstrated 80 that careful selection of the hydrodynamic boundary conditions in a planar flow-focusing device can 81 enable production of microbubbles with dimensions one order of magnitude smaller than the

82 microchannel width, when the length of the exit channel is designed to be significantly greater than

83 its width (Castro-Hernández, et al. 2011). With this method, microbubbles $\sim 5 \mu \mathrm{m}$ diameter and with

84 a polydispersity index $<5 \%$ could be produced at a rate $>10^{5}$ microbubbles $/$ sec, which represents an

85 improvement compared to more conventional microfluidic systems based on microbubble pinch-off.

86 However, manufacturing of the microchannels in this study still required costly and time-consuming

87 photolithographic techniques, and scaling up production of microbubble suspensions with a mean

88 diameter of $1-3 \mu \mathrm{m}$ (often employed in therapeutic applications) could pose challenges.

89 Identifying a microbubble production method that relies on further scaled-up channel

90 architectures (i.e., up to $100 \mathrm{~s}$ or $1000 \mathrm{~s}$ of $\mu \mathrm{m}$ ) would enable both greater production rates and

91 overcome challenges associated with clogging, high backpressure and manufacturing costs. However,

92 additional modifications to the microbubble production mechanism or the physico-chemical

93 properties of fluidic environment may be required in order to produce microbubbles of clinically 
94 relevant sizes via these scaled-up devices. For instance, devices consisting of off-the-shelf capillaries

95 embedded within an easy-to-fabricate T-junction manifold have been previously employed to produce 96 microbubbles (Parhizkar, et al. 2013, Parhizkar, et al. 2015, Parhizkar, et al. 2014). Parhizkar et al.

97 employed capillaries with inner diameter in the range 100-200 $\mu \mathrm{m}$, and investigated the effect of

98 varying the viscosity and surface tension of the liquid phase on microbubble size and size distribution

99 (Parhizkar, et al. 2013). They showed that bubble diameter could be reduced down to approximately

100 half of the capillary diameter, but that the minimum bubble diameter $(\sim 50 \mu \mathrm{m})$ was still too large for

101 clinical use. Moreover, addition of viscosity-enhancers should be considered carefully in clinical 102 formulations.

C. Multi-stage and hybrid devices for microbubble production

Approaches relying on a two-stages microbubble production process have also been proposed,

105 whereby larger precursor bubbles are produced in a first step, and their size is subsequently reduced

106 down to clinically-applicable levels in a second step. Given the larger size of the precursor bubbles, it

107 is possible to utilise channels of larger dimensions compared to microfluidic devices relying on a

108 single-step microbubble production process. For example, Gnyawali et al. employed a $20 \mu \mathrm{m}$ flow

109 focusing orifice to produce bubbles of $\sim 100 \mu \mathrm{m}$ diameter, which were then conveyed through a

110 serpentine shaped channel around which a negative pressure was applied (Gnyawali, et al. 2017). As

111 the bubbles traversed this channel, the generated vacuum drove gas out of the bubbles, which in turn

112 shrunk down to a useable clinical range of 1-7 $\mu \mathrm{m}$ in diameter. Microbubbles were stable at

113 atmospheric pressure for at least 25 minutes, although their acoustic response or handling stability

114 were not reported. Additionally, as each large precursor bubble generates only a single microbubble,

115 the microbubble concentration in the end-product is likely to be low. Finally, the gas within the

116 microbubbles may be irreversibly lost without some form of scavenging, which may be problematic if

117 an expensive and/or polluting gas (e.g., sulphur hexafluoride) is employed as the microbubble core. 
119 sonication of precursor bubbles induced by low-frequency ultrasound waves. Its implementation has

120 been reported in a study by Chen et al. Large gas bubbles were firstly conveyed through an $860 \mu \mathrm{m}$

121 (inner diameter) polyethylene tube to form a gas-in-liquid slug flow regime, with the fluid containing

$122 \sim 100-300 \mathrm{~nm}$ diameter ethyl cellulose particles. They subsequently travelled in front of a $20 \mathrm{kHz}$

123 ultrasonic horn, externally coupled to the capillary, causing cavitation to occur at the interface between

124 the gas bubbles and the particle-rich fluid. Cavitation resulted in the formation of microbubbles, which

125 were stabilised by adsorption of the nanoparticles onto the gas-liquid interface. microbubble size

126 dispersity could be reduced by increasing the sonication power, but it still encompassed a broad

127 diameter range (i.e. from approximately 5 to $60 \mu \mathrm{m}$ ) (Chen, et al. 2014). The microbubble size

128 distribution with the lowest dispersity had a peak diameter of $\sim 20 \mu \mathrm{m}$, which is beyond the accepted

129 limit for intravenous administration. Furthermore, the fluid temperature was observed to increase up

130 to $80^{\circ} \mathrm{C}$ within 7 minutes of sonication, which may hinder the applicability of this method to the

131 production of microbubbles loaded with bioactive compounds. However, compared to the approach

132 by (Gnyawali, et al. 2017), this method enables production of multiple microbubbles from a single

133 precursor bubble and therefore presents greater scalability potential. A summary of the microbubble

134 sizes, polydispersity and production rates reported for published device is shown in Table 1 to show

135 the key advances in size control and/or production rate.

136 Ohl et al. developed a T-junction microfluidic device coupled with a piezoelectric element, to

137 investigate the behaviour of a gas-liquid interface exposed to $\sim 100 \mathrm{kHz}$ continuous ultrasound waves.

138 It was shown that these interfaces develop standing surface waves, the amplitude of which depended

139 upon the driving acoustic intensity (Ohl, et al. 2010). Pronounced crests formed at sufficiently high

140 intensity, resulting in the entrapment of small bubbles between neighbouring and coalescing crests.

141 These bubbles in turn underwent inertial cavitation and fragmentation; however, their size was not 
142 fully characterised. The study thus demonstrates that it is possible to design a microfluidic system

143 coupled with an ultrasound source, in which the amplitude of the ultrasound wave can modulate the

144 surface oscillation of larger precursor bubbles, potentially leading to the formation of smaller

145 micrometre-scale bubbles. It also demonstrates that, by designing the system to maximise the acoustic

146 energy within the microfluidic channels, heat losses can be minimised. A similar approach however,

147 has not yet been investigated for the production of coated microbubbles with clinically relevant

148 characteristics.

149 The aim of the present study was therefore to determine whether a hybrid sonication-

150 microfluidic (or 'sonofluidic') device could be used to produce microbubbles in the 1-2 $\mu$ m diameter

151 range with a clinically acceptable size distribution (all bubbles $<5 \mu \mathrm{m}$ ), and with production rates and

152 microbubble stability comparable to those of batch methods.

\begin{tabular}{|c|c|c|c|c}
\hline $\begin{array}{c}\text { Approximate } \\
\text { MB mean } \\
\text { diameter } \\
(\boldsymbol{\mu} \mathbf{m})\end{array}$ & $\begin{array}{c}\text { Polydispersity } \\
\text { index } \\
\text { immediately } \\
\text { after } \\
\text { manufacture }\end{array}$ & $\begin{array}{c}\text { Maximum MB } \\
\text { Production } \\
\text { Rate } \\
(\mathbf{M B s} / \mathbf{s e c})\end{array}$ & $\begin{array}{c}\text { Primary MB } \\
\text { shell constituent }\end{array}$ & Reference \\
\hline \hline 5 & $<\mathbf{2 \%}$ & $10^{6}$ & Phospholipid & Hettiarachchi et al. 2007 \\
\hline \hline 4 & $<\mathbf{2 \%}$ & $10^{6}$ & $\begin{array}{c}\text { Phospholipid and } \\
\text { protein }\end{array}$ & Seo et al. 2010 \\
\hline \hline 2 & $10-50 \%$ & $10^{6}$ & Phospholipid & Peyman et al. 2012 \\
\hline \hline 5 & Not cited & Not cited & Phospholipid & Gnyawali et al. 2017 \\
\hline \hline 2.5 & $3 \%$ & $10^{6}$ & Phospholipid & van Elburg et al. 2021 \\
\hline
\end{tabular}

153

154

155

156

157
Table 1: Summary of the microbubble sizes, polydispersity and production rates reported for selected published microfluidic devices. 


\section{MATERIALS AND METHODS}

\section{A. Sonofluidic device for microbubble production}

\section{Operating principle}

The sonofluidic device developed in this study relies on a two-stages process to produce coated

163 gas microbubbles, as illustrated in Figure 1A. In the first stage, relatively large bubbles $(\sim 180 \mu \mathrm{m}$ in

164 diameter) with a narrow size distribution are generated from a simple T-junction channel architecture

165 by a hydrodynamic pinch-off mechanism. In the second stage, bubbles are exposed to low-frequency

166 ultrasound from a piezoelectric transducer coupled with the exit channel from the T-junction, which

167 causes the large bubbles to 'release' a population of smaller microbubbles (with mean diameter

168 typically of $\sim 1-2 \mu \mathrm{m})$. This operating principle was selected on the basis of the following postulated

169 benefits compared to more conventional microfluidic-based approaches: (i) multiple microbubbles

170 can be produced from a single precursor bubble to enhance production rates; (ii) high acoustic energy

171 density can be generated within the microfluidic channel whilst minimising heat losses, which enables

172 both effective absorption of coating material at the gas-liquid interface as well as incorporation of

173 thermolabile bioactive compounds; (iii) given the relatively large size of the precursor bubbles, the

174 microfluidic channels can be manufactured using cost-effective, easy-to-perform, and scalable

175 techniques, and devices can be operated at larger volumetric flow rates; and (iv) devices can be

176 potentially integrated with detection systems for in-line quantification of microbubble size

177 distribution. 

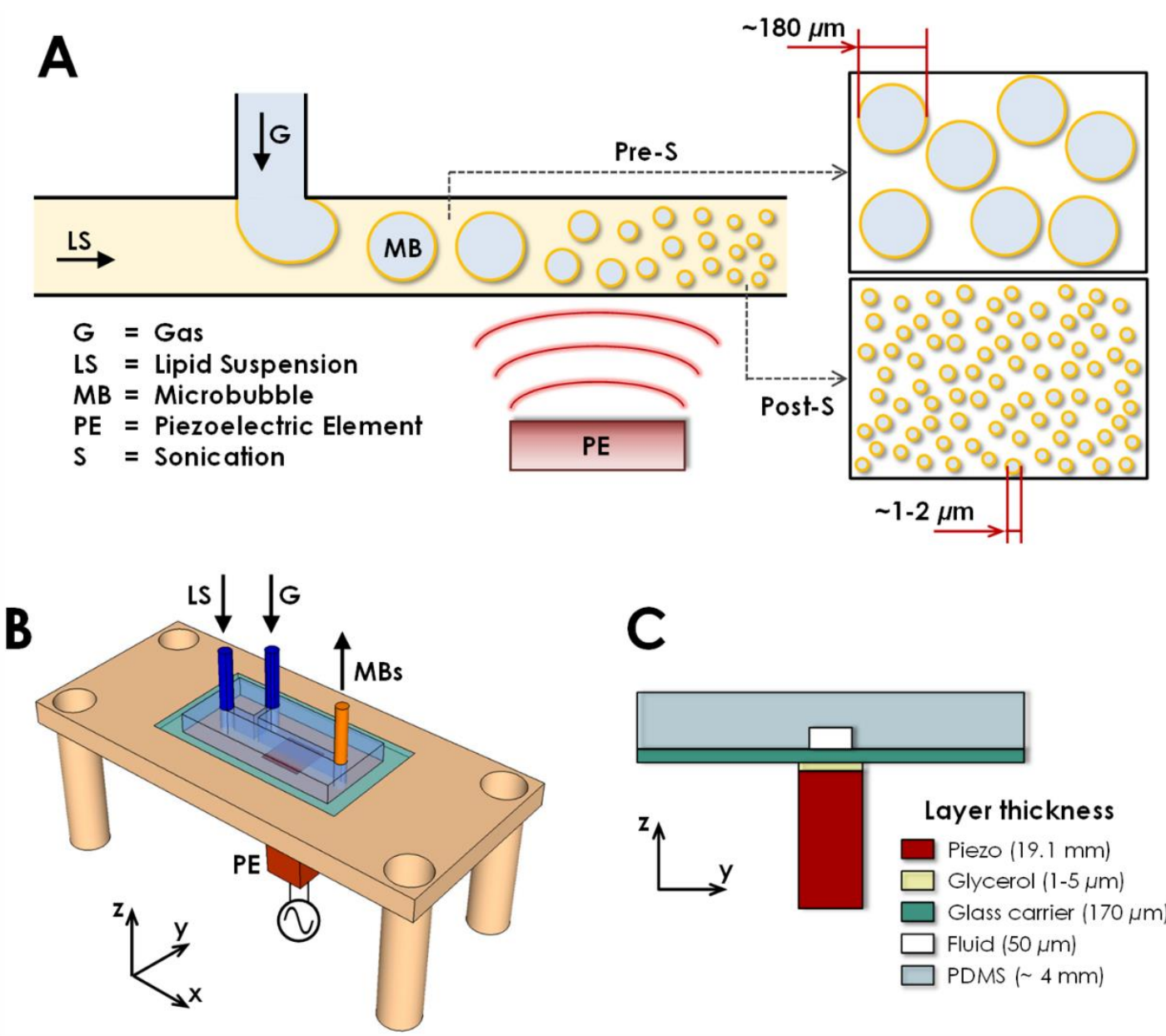

179 FIG. 1. (A) Schematic depiction of the operating principle governing microbubble generation within

180 the sonofluidic device. Larger bubbles $(\sim 180 \mu \mathrm{m}$ in diameter $)$ are produced using a $\mathrm{T}$-junction

181 microfluidic architecture and exposed to a low frequency (in the range $71-73 \mathrm{kHz}$ ) ultrasound field,

182 causing the precursor bubbles to release smaller microbubbles (with mean diameter of $\sim 1-2 \mu \mathrm{m}$ ). (B)

183 Schematic depiction of the overall sonofluidic device assembly. The microfluidic T-junction device

184 was positioned on to a custom-built holder, and coupled with a piezolectric element (PE) generating

185 the ultrasound field. Flows of gas $(\mathrm{G})$ and a lipid suspension (LS) were conveyed through the device

186 inlets, while the generated microbubble (microbubble) suspension was collected from the outlet. (C)

187 Cross-sectional view of the constitutive layers of the sonofluidic device, with indicated the 188 corresponding thickness for each layer. The ultrasound field was generated by a $19.1 \mathrm{~mm}$ thick 
189 piezoelectric element (piezo), and travelled through a $0.17 \mathrm{~mm}$ thick glass carrier layer that was coupled

190 to the piezoelectric transducer via a thin layer of glycerol (estimated thickness: $1-5 \mu \mathrm{m})$. It then

191 propagated into the fluid layer of the microfluidic device (thickness: $50 \mu \mathrm{m}$ ), and subsequently through

$192 \mathrm{a} \sim 4 \mathrm{~mm}$ thick layer of PDMS.

\section{Device design, manufacturing and assembly}

194

The microfluidic T-junction comprised two inlet and one outlet channels, with a rectangular cross-

section (width $\times$ depth) of $250 \mu \mathrm{m} \times 50 \mu \mathrm{m}$ (liquid suspension inlet and outlet) and $125 \mu \mathrm{m} \times 50 \mu \mathrm{m}$

196 (gas inlet). This channel architecture was cast in poly(dimethylsiloxane) (PDMS, Sylgard ${ }^{\mathrm{TM}}$ 184, Dow

197 Inc., Michigan, USA) using a combined micromilling-replica moulding ( $\mu$ Mi-REM) technique

198 described previously (Carugo, et al. 2016). Briefly, the channel architecture was micromilled into a

199 poly(methyl methacrylate) (PMMA, theplasticshop.co.uk, Coventry, UK) block to form a negative

200 mould. A positive mould was manufactured by coating the milled PMMA mould with a bi-component

201 epoxy adhesive (1:1 weight ratio between components, Yellow Dual Cartridge, RS Components Ltd.,

202 Corby, UK), which was then degassed by vacuum to remove entrapped air bubbles and left to cure at

203 room temperature. After curing, the positive epoxy mould was removed from the PMMA block, and

204 a 10:1 (w/w) mixture of PDMS and curing agent was poured over it and degassed for approximately

20530 minutes to remove entrapped air bubbles. The PDMS layer was then cured overnight at room

206 temperature. To complete the manufacturing process, the PDMS layer was removed from the positive

207 epoxy mould and the patterned surface was activated by plasma treatment (using a plasma cleaner

208 ATTO, Diener electronic GmbH, Ebhausen, Germany) along with a $74.00 \mathrm{~mm} \times 49.00 \mathrm{~mm} \times 0.17$

209 mm (length $\times$ width $\times$ thickness) glass layer (Logitech, Glasgow, UK). After $\sim 60-80$ s of treatment,

210 the PDMS layer was pressed firmly against the glass, and the assembly was heat treated on a hotplate

211 set to $100^{\circ} \mathrm{C}$ for 10 minutes to achieve effective bonding between glass and PDMS. To create inlet

212 and outlet ports for the gas and liquid flows, 1/16" polyether ether ketone (PEEK) rods were glued 
213 by low-cost solvent-free glue (Pritt, Henkel Ltd., Herts., UK) onto the epoxy layer before PDMS

214 pouring. The rods were then removed upon PDMS curing, prior to plasma treatment. After bonding

215 to the glass surface, short segments of 3/32" OD Tygon ${ }^{\circledR}$ tubing (Cole-Parmer Instrument Co. Ltd.,

216 London, UK) were inserted into the ports to act as connectors for $1 / 16$ " OD tubing. These were

217 connected to relevant syringes or gas circuits by 18G blunt needles (Sigma Aldrich, Gillingham, UK).

218 The PDMS device was then placed on to a custom holder (manufactured from polyoxymethylene),

219 which contained a recess, in which the microfluidic device was positioned, and a central cut out

220 window through which an ultrasound transducer was placed for coupling with the glass layer of the

221 device (Figure 1B). Reversible coupling was achieved using a small volume of glycerol, which allowed

222 for easy removal or replacement of the microfluidic device when required. The thin glass layer thus

223 acted as an effective carrier for the ultrasound wave generated by the transducer, which was positioned

$2245 \mathrm{~mm}$ away from the junction between inlet channels. PDMS has a comparable characteristic acoustic

225 impedance to the one of water (Carugo, et al. 2015, Leibacher, et al. 2014), it was therefore anticipated

226 that ultrasound reflections at the liquid-PDMS interface would be minimised. The ultrasound

227 transducer consisted of a single piezoelectric element $(9.0 \mathrm{~mm} \times 9.0 \mathrm{~mm} \times 19.1 \mathrm{~mm}, \mathrm{Pz} 26$, Meggitt

228 PLC, UK) with a fundamental thickness resonance frequency of $69 \mathrm{kHz}$. Figure $1 \mathrm{C}$ shows a cross-

229 sectional view of the constitutive layers of the sonofluidic device, in the region where the transducer

230 is coupled with the glass layer, with the corresponding thickness values indicated for each layer.

231 3. Electronic components and ultrasound actuation methods

232 The transducer was driven in continuous mode by a $55 \mathrm{~dB}$ RF power amplifier (1040L, E\&I, 233 Rochester, NY, USA) fed from a signal generator (Agilent 33220A, Keysight Technologies, Santa

234 Rosa, USA). Two different actuation methods were used in the present study, namely single frequency

235 (SF) and frequency modulation (FM). In the latter method, a linear frequency sweeping was applied, 
236 and the effects of varying both the frequency range and sweep period (or duration) on microbubble

237 characteristics were investigated.

\section{B. Microbubble Formulations}

239 Phospholipids were selected as the primary microbubble coating material in this study, as they are the

240 most commonly used shell constituent in commercial contrast agents (e.g., SonoVue ${ }^{\circledR}$, Sonazoid $\mathbb{}$ 241 and Definity®) (Frinking, et al. 2020). The lipids 1,2-distearoyl-sn-glycero-3-phosphocholine (DSPC,

242 850365), 1,2-dipalmitoyl-sn-glycero-3-phosphocholine (DPPC, 850355) 1,2-Dipalmitoyl-sn-glycero-

243 3-phosphatidic acid sodium salt (DPPA, 830855), 1,2-distearoyl-sn-glycero-3-phosphoethanolamine-

244 N-[methoxy(polyethylene glycol)-5000] (DSPE-mPEG5000, 880220) and 1,2-dipalmitoyl-sn-glycero-

245 3-phospho-(1'-rac-glycerol) (DPPG, 840455) were purchased as a $25 \mathrm{mg} / \mathrm{mL}$ solution in chloroform

246 or powders from Avanti Polar Lipids, Inc. (Alabaster, AL, USA). Two different formulations of the

247 microbubble shell were investigated, corresponding to: (i) a mixture of DSPC and polyoxyethylene

248 (40) stearate (PEG-40S), which is a composition widely used in research settings (Borden, et al. 2005,

249 Owen, et al. 2018); and (ii) a mixture of DPPC, DSPE-mPEG5000, and DPPA, which is comparable

250 to the formulation of the clinically approved Definity® microbubbles (Lantheus Medical Imaging,

251 MA, USA) (Segers, et al. 2017). In the first formulation, DSPC (25 mg/mL in chloroform) and PEG-

$25240 \mathrm{~S}(10 \mathrm{mg} / \mathrm{mL}$ in chloroform) were mixed in a glass vial to form a chloroform solution at a molar

253 ratio of 9:1, respectively. In the second formulation, DPPC (25 mg/mL in chloroform), DSPE-

$254 \mathrm{mPEG} 5000(25 \mathrm{mg} / \mathrm{mL}$ in chloroform $)$ and DPPA $(1 \mathrm{mg} / \mathrm{mL}$ in a chloroform, methanol and water

255 mix) were mixed in a glass vial to a $20 \mathrm{mg}$ total of lipid constituents at a molar ratio of 8:1:1,

256 respectively. Chloroform solutions were covered with perforated Parafilm ${ }^{\circledR}$ (Bemis Company, Inc.,

257 Neenah, WI, USA) and allowed to evaporate overnight to form a homogenous lipid film. $10 \mathrm{~mL}$ Milli-

258 Q water (Merck Millipore, Watford, UK) or a water, glycerol and propylene glycol mixture (80:10:10

$259 \mathrm{v} / \mathrm{v}$ respectively) was added to the DSPC or Definity ${ }^{\circledR}-$ like lipid films, respectively. In the case of the 
DSPC-based formulation, three different DSPC concentrations in the final suspension were

261 investigated, corresponding to 2,4 and $6 \mathrm{mg} / \mathrm{mL}$, to assess whether lipid concentration had an effect

262 on the characteristics of microbubbles produced using the sonofluidic device. The lipids were

263 resuspended into the solvent by stirring at $100^{\circ} \mathrm{C}$ on a magnetic stirrer hotplate for a minimum of 30

264 minutes. They were then homogeneously dispersed within the solution by sonication for

265 approximately 2.5 minutes using a micro-sonicator tip fully immersed in the solution at a power setting

266 of 2 to 3 (Microson XL 2000, QSonica, Newtown, CT, USA).

\section{Experimental procedures}

268 Microbubbles produced using the sonofluidic device were compared with those produced by

269 conventional sonication in terms of their size, stability, and concentration. In addition, the effect of

270 changing the sonofluidic device driving ultrasound parameters was investigated, and performance

271 consistency across multiple devices was assessed. Finally, different lipid formulations and

272 concentrations were investigated.

\section{Production of lipid microbubbles by conventional sonication}

274 After resuspension and dispersion of lipids into the solvent, the sonicator tip was placed at the

275 air-liquid interface and the headspace in the vial filled with nitrogen gas. The solution was sonicated

276 under constant nitrogen flow for 30 seconds at a power setting of 14, to form a suspension of

277 microbubbles. The suspension was left to cool to room temperature over 5 minutes. Typically, clinical

278 microbubble formulations use heavy gases such as perfluorobutane or sulfur hexafluoride, but to

279 facilitate a large number of experiments, nitrogen (supplied by BOC Gases, Guildford, UK) was used 280 in this study. 
The device channels were flushed with ethanol and deionised water multiple times prior to use.

283 The resuspended, fully dispersed lipid solution was transferred to a $10 \mathrm{~mL}$ syringe and connected to

284 the liquid inlet port of the device. The gas inlet was connected to a nitrogen cylinder via a dual stage

285 regulator with cut-off valve and an inline electronic pressure manometer (2023P Digitron, Elektron

286 Technology, Cambridge, UK). A syringe pump (World Precision Instruments Inc., Florida, USA) was

287 used to vary lipid flow rates into the device, whilst gas pressure control was supplied by the regulator.

288 The device was run for one minute to establish a stable 'pinch-off regime at the T-junction, indicated

289 by the appearance of a steady stream of large bubbles. This regime was achieved at a volumetric flow

290 rate of the lipid suspension of $0.5 \mathrm{~mL} / \mathrm{min}$ and a nitrogen inlet pressure of $37 \mathrm{kPa}$. The mean diameter

291 of the produced bubbles was approximately $180 \mu \mathrm{m}$, determined through optical microscopy of the

292 collected bubble suspension (please see below). Upon formation of a steady bubble flow, the

293 ultrasound transducer was actuated, which resulted in the production of smaller microbubbles. These

294 were also collected from the outlet tube for microscopic analysis.

\section{Microbubble concentration, size and stability analysis}

296 Sonicated microbubbles were homogeneously dispersed by gentle manual agitation of the vial, and

$29710 \mu \mathrm{L}$ of the suspension were loaded on a coverslip-covered haemocytometer via a pipette. The 298 continuous-flow format of the sonofluidic device meant that the outlet tube could be directly 299 connected to the hemocytometer chamber. Microbubbles were imaged using a brightfield microscope 300 (Leica Microsystems GmbH, Wetzlar, Germany), and images were acquired using a digital camera 301 (MicroPublisher 3.3 RTV, QImaging, Surrey, Canada). A 4× or 40× objective was used for imaging 302 the larger precursor bubbles $(\sim 180 \mu \mathrm{m}$ mean diameter $)$ and smaller microbubbles $(\sim 1-10 \mu \mathrm{m}$ 303 diameter), respectively. Microbubble size and concentration were determined using a purpose-written 304 image processing program in MATLAB (The Mathworks Inc., Natick, MA, USA), as previously 305 described in Sennoga et al. (Sennoga, et al. 2012). A minimum of twenty images were analysed for each 
sample. For stability analysis, microbubble size and concentration were measured as described every

30710 minutes from the same sample. This was repeated three times using a fresh bubble suspension

308 created from a new lipid film each time. The average microbubble mean diameter values reported in

309 this manuscript are accompanied by the corresponding average standard deviation of the diameter

310 distribution, which provides a quantification of microbubble size dispersity. Experiments were

311 performed at the ambient room temperature $\left(\sim 18-22^{\circ} \mathrm{C}\right)$ and pressure. The microscope lamp was

312 switched off in between measurements to avoid excessive heating of the sample, and the coverslip

313 was left in place on the haemocytometer throughout.

\section{Optimisation of sonofluidic device operating parameters}

315 The effects of varying the sonofluidic device operating ultrasound parameters on microbubble

316 production rate and size distribution were investigated. Parameters included the acoustic energy (e.g.,

317 by changing the input driving voltage to the amplifier in the range $300-900 \mathrm{mV}$ ), the frequency value

318 in single frequency operation mode (in the range $67-76 \mathrm{kHz}$ ), and the sweep frequency range

319 (between $69 \mathrm{kHz}$ and $73 \mathrm{kHz}$ ) and period (in the range $1-1000 \mathrm{~ms}$ ) in frequency modulation mode.

320 Optimal operating parameters were identified, which provided the greatest production rate whilst

321 retaining a clinically relevant mean microbubble diameter (of $\sim 1-2 \mu \mathrm{m}$ ) and a low size dispersity.

\section{Reproducibility of the sonofluidic device}

323 To test the performance reproducibility of the sonofluidic device, three devices of the same design

324 were constructed and run at the optimal ultrasound settings. Microbubbles in these experiments were 325 produced using three independent lipid suspensions of DSPC:PEG40s (9:1 molar ratio).

327 A modified design of the sonofluidic device was developed to perform a preliminary investigation 328 of whether microbubble production rates could be scaled-up by increasing both the microfluidic 
329 device cross-sectional dimensions (and thus the operating flow rates) and the ultrasound field intensity.

330 This scaled-up sonofluidic device was designed, manufactured, and operated following the same

331 principles and procedures reported above. The ultrasound transducer was changed to a $60 \mathrm{kHz}$

332 piezoelectric element (maximum power: $30 \mathrm{~W}$, PZT-4, Beijing Ultrasonic, China), and the glass layer

333 to a thicker $75 \mathrm{~mm} \times 25 \mathrm{~mm} \times 1 \mathrm{~mm}$ (length $\times$ width $\times$ thickness) slide to effectively sustain the

334 greater levels of ultrasound-induced strain. The fluidic channels of the T-junction architecture had

335 increased cross-sectional dimensions (width $\times$ thickness) of $1.0 \times 0.1 \mathrm{~mm}$ (liquid suspension inlet and

336 exit channels) and $0.5 \times 0.1 \mathrm{~mm}$ (gas inlet channel). In the experiments using this scaled-up device,

337 the ultrasonic transducer was driven at a peak-to-peak voltage (post amplification) of $200 \mathrm{~V}$ and the

338 driving frequency was linearly swept between 60 and $62 \mathrm{kHz}$. These ultrasound settings were

339 maintained constant throughout the experiments, while the volumetric flow rate of the lipid

340 suspension was increased from 5 to $35 \mathrm{~mL} / \mathrm{min}$. The gas inlet pressure was manually adjusted as the

341 liquid flow rate was increased, in order to maintain a stable stream of precursor bubbles forming at

342 the junction between inlet channels. The lipid suspension in these experiments comprised DSPC and

343 PEG40S at a molar ratio of 9:1, suspended in a water, glycerol and propylene glycol mixture (80:10:10

$344 \mathrm{v} / \mathrm{v}$ respectively). The microbubbles size and concentration were determined by optical microscopy,

345 following the protocol described above.

346 III. RESULTS AND DISCUSSION

347 A. Optimisation of sonofluidic device operating parameters

348 Figure 2 shows representative diameter distributions of phospholipid-coated (DSPC:PEG40S)

349 precursor bubbles (i.e., not exposed to the ultrasound field) and the microbubbles generated upon

350 activation of the piezoelectric transducer. Precursor bubbles formed at the T-junction by a pinch-off

351 mechanism, and had a mean diameter of $181 \mu \mathrm{m}$ and standard deviation of the distribution of $34 \mu \mathrm{m}$

352 (Figure 2A), corresponding to a polydispersity index (PDI) of 0.035. Once exposed to the ultrasound 
353 field, they formed microbubbles with a size distribution having mean diameter of $1.45 \mu \mathrm{m}$ and 354 standard deviation of $0.76 \mu \mathrm{m}$ (Figure 2B), corresponding to a PDI of 0.27 .
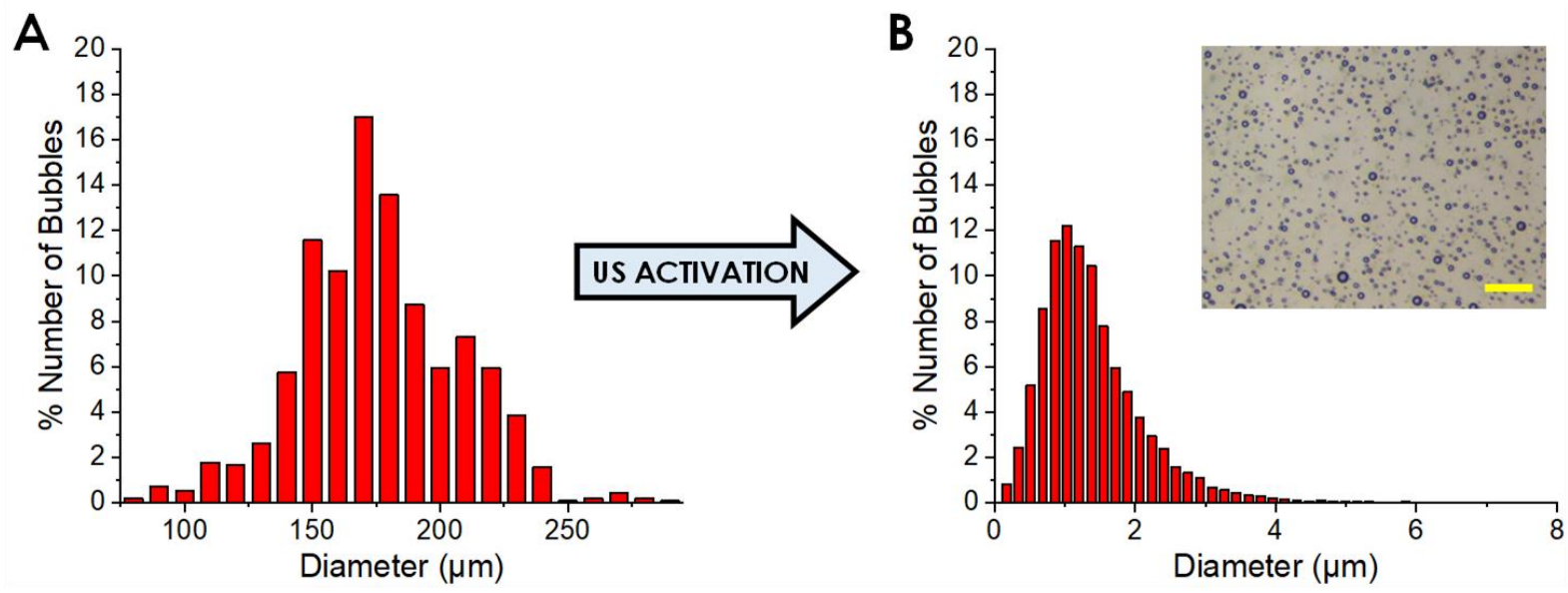

FIG. 2. Representative percentage weighted size distributions of (A) bubbles prior to activation of the

357 piezoelectric transducer (based on 20 images of $\sim 1000$ bubbles), and (B) microbubbles generated 358 upon activation of the ultrasound field (based on 30 images of $\sim 13000$ bubbles). The inset shows a 359 representative microscope image of microbubbles produced using the sonofluidic device (scale bar: $36010 \mu \mathrm{m})$.

In order to determine the optimal operating parameters for the device, the driving ultrasound

362 frequency, amplitude, and frequency sweep settings were all varied. In a first series of experiments, 363 the transducer was driven in SF mode, and the effect of varying the ultrasound frequency was 364 investigated. Frequency values evaluated were equal to 67, 69, 71, 73 and $76 \mathrm{kHz}$, at a constant pre365 amplifier input voltage of $900 \mathrm{mV}$ (see Figure 3A). It was found that increasing the ultrasound 366 frequency from $67 \mathrm{kHz}$ to $69 \mathrm{kHz}$ resulted in an increase in microbubble concentration (from $0.43 \pm$ $3670.06 \times 10^{8}$ microbubbles $/ \mathrm{mL}$ to $1.05 \pm 0.33 \times 10^{8}$ microbubbles $/ \mathrm{mL}$ ) and a slight decrease in 368 microbubble mean diameter and size dispersity (from $2.19 \pm 1.28 \mu \mathrm{m}$ to $1.90 \pm 1.02 \mu \mathrm{m}$ ). A similar 369 trend was observed when the frequency was further increased from $69 \mathrm{kHz}$ to $71 \mathrm{kHz}$ (microbubble 370 concentration: $1.99 \pm 0.25 \times 10^{8}$ microbubbles $/ \mathrm{mL}$; mean microbubble diameter: $\left.1.56 \pm 0.83 \mu \mathrm{m}\right)$. 
371 However, microbubbles produced at $73 \mathrm{kHz}$ had comparable mean diameter and size dispersity (1.54

$372 \pm 0.79 \mu \mathrm{m})$ to those produced at $71 \mathrm{kHz}$, and only a slightly reduced concentration $\left(1.69 \pm 0.29 \times 10^{8}\right.$

373 microbubbles $/ \mathrm{mL}$ ). Further increasing the driving frequency to $76 \mathrm{kHz}$ caused a marginal increase in

374 both microbubble size (mean diameter: $1.90 \pm 1.35 \mu \mathrm{m})$ and concentration $\left(1.88 \pm 0.49 \times 10^{8}\right.$

375 microbubbles $/ \mathrm{mL}$ ), but this was accompanied by an increase in the corresponding standard

376 deviations. In a second series of experiments, the transducer was operated by applying a linear

377 frequency modulation over a range of driving frequencies that excluded those values that resulted

378 either in the lowest microbubble production rate $(67 \mathrm{kHz})$ or the greatest variability in microbubble

379 concentration and size $(76 \mathrm{kHz})$. The sweep frequency ranges investigated were $69-73 \mathrm{kHz}, 70-72$

$380 \mathrm{kHz}$, and $71-73 \mathrm{kHz}$, at a constant sweep period of $50 \mathrm{~ms}$. Among the different modulation regimes

381 evaluated, the $71-73 \mathrm{kHz}$ sweep range resulted in the greatest microbubble concentration $(3.22 \pm 0.69$

$382 \times 10^{8}$ microbubbles $\left./ \mathrm{mL}\right)$, corresponding to a production rate of approximately $2.7 \times 10^{6}$

383 microbubbles/s, and a clinically viable mean diameter of $1.45 \pm 0.79 \mu \mathrm{m}$, and was thus selected as the

384 preferred operating condition for subsequent tests. Operating the device in frequency sweeping also

385 brings with it additional benefits, such as reduced temperature sensitivity, less requirements for

386 controlling the ultrasound frequency (i.e., through automated frequency tracking methods), and

387 potentially greater uniformity of the acoustic field within the fluid layer, as previously reported for

388 other acoustofluidic devices (Carugo, et al. 2014, Manneberg, et al. 2009). It should be noted that the

389 optimal driving frequencies did not include the nominal resonance frequency of the piezoelectric

390 element $(67 \mathrm{kHz})$, which could be due to the effect of coupling the transducer with the microfluidic

391 device structure. In some cases, it could also be observed that the produced microbubbles weren't

392 efficiently released from the device, potentially due to the 'trapping' effect of acoustic radiation forces.

393 Future studies could thus evaluate a wider range of combinations of driving frequencies and inlet 
394 volumetric flow rates, as well as the applicability of a pulsed ultrasound mode, to further optimise 395 microbubbles production rate.

397 investigated (see Figure 3B), at a frequency sweep range of $71-73 \mathrm{kHz}$ and period of $50 \mathrm{~ms}$. By varying 398 the pre-amplifier input voltage from $300 \mathrm{mV}$ to $500 \mathrm{mV}$ resulted in a significant increase in 399 microbubble concentration (from $0.82 \pm 0.16 \times 10^{6}$ to $0.52 \pm 0.39 \times 10^{8}$ microbubbles $/ \mathrm{mL}$ ) and a 400 reduction in microbubble mean diameter and corresponding standard deviation (from $4.39 \pm 2.41 \mu \mathrm{m}$ 401 to $2.06 \pm 1.13 \mu \mathrm{m})$. When the input voltage was further increased to $700 \mathrm{mV}$, it was again observed 402 an increase in microbubble concentration $\left(1.81 \pm 0.21 \times 10^{8}\right.$ microbubbles $\left./ \mathrm{mL}\right)$ and a corresponding 403 reduction in both microbubble mean diameter and standard deviation $(1.63 \pm 0.89 \mu \mathrm{m})$. The observed 404 effect of the ultrasound intensity on microbubble characteristics may be due to the fact that the gas405 liquid interface of precursor bubbles underwent oscillations of greater amplitude at the higher 406 ultrasound intensities, which resulted in more frequent entrapments and subsequent fragmentation 407 events of smaller microbubbles, consistently with the observations by Ohl and co-authors (Ohl, et al. 408 2010). The relationship between driving voltage and microbubble properties was however non-linear, 409 and a further increase in the pre-amplifier input voltage (up to $900 \mathrm{mV}$ ) did not cause significant 410 changes in both microbubble concentration $\left(1.65 \pm 0.54 \times 10^{8}\right.$ microbubbles $\left./ \mathrm{mL}\right)$ and size $(1.69 \pm$ $4110.87 \mu \mathrm{m})$. Whilst input voltages of $700 \mathrm{mV}$ and $900 \mathrm{mV}$ led to comparable microbubble characteristics,

412 the latter was selected as the preferred operating voltage in order to accommodate for potential 413 reductions in the acoustic energy within the device, i.e., due to fluctuations in environmental 414 temperature, variabilities in the manufacturing process, or other factors. Results also suggest that 415 varying the input voltage to the sonofluidic device may be an effective way of tuning the microbubble 416 mean diameter, although careful consideration should be given to the corresponding reduction in 417 microbubble concentration. 
419 Figure 3C). The transducer was driven at a pre-amplifier input voltage of $900 \mathrm{mV}$, and the frequency 420 was linearly swept between 71 and $73 \mathrm{kHz}$ over a period of either 1, 5, 50, 250, 500, and $1000 \mathrm{~ms}$.

421 Interestingly, the highest microbubble concentrations were generated at $1 \mathrm{~ms}$ and $1000 \mathrm{~ms}$ sweep

422 duration $\left(1.66 \pm 0.81 \times 10^{8}\right.$ microbubbles $/ \mathrm{mL}$ and $2.52 \pm 0.35 \times 10^{8}$ microbubbles $/ \mathrm{mL}$, respectively),

423 with a corresponding microbubble diameter of $1.73 \pm 0.83 \mu \mathrm{m}(1 \mathrm{~ms})$ and $1.45 \pm 0.76 \mu \mathrm{m}(1000 \mathrm{~ms})$.

424 Although there was no obvious relationship between microbubble characteristics and sweep period,

425 the greater production rate at the lowest sweep period $(1 \mathrm{~ms})$ may be due to the fact that precursor

426 bubbles were exposed to the optimal operating frequency for a longer period of time as they travelled

427 above the transducer, when compared to greater sweep periods (5 - $500 \mathrm{~ms}$ ). The reasons behind

428 increased microbubble production rates at the highest sweep period $(1000 \mathrm{~ms})$ are not fully understood

429 yet, and merit further investigations. Previous studies using acoustofluidic resonators have shown that

430 frequency modulation can induce an oscillatory translational motion on particles or biological cells

431 suspended in a microfluidic cavity, and that the amplitude of oscillation scales with the sweep duration

432 (Ankrett, et al. 2013, Jonnalagadda, et al. 2018). It may thus be hypothesised that this enhanced

433 oscillatory motion may promote release of microbubbles from the gas-liquid interface of precursor

434 bubbles, overall resulting in greater microbubble concentrations in the end-product. Future studies

435 using ultra-high speed microscopy will be performed to gain a more pervasive understanding of the

436 effect of sweep duration on precursor bubble behaviour and microbubbles production mechanism.

437 Overall, the sonofluidic device operating parameters were chosen to be: $71-73 \mathrm{kHz}$ frequency

438 sweep over $1000 \mathrm{~ms}$, at an input pre-amplification voltage of $900 \mathrm{mV}$, producing microbubbles of

439 mean diameter of $\sim 1.45 \mu \mathrm{m}$ and PDI $=0.27$, at a production rate in the order of $\sim 2 \times 10^{6}$

440 microbubbles/s. 

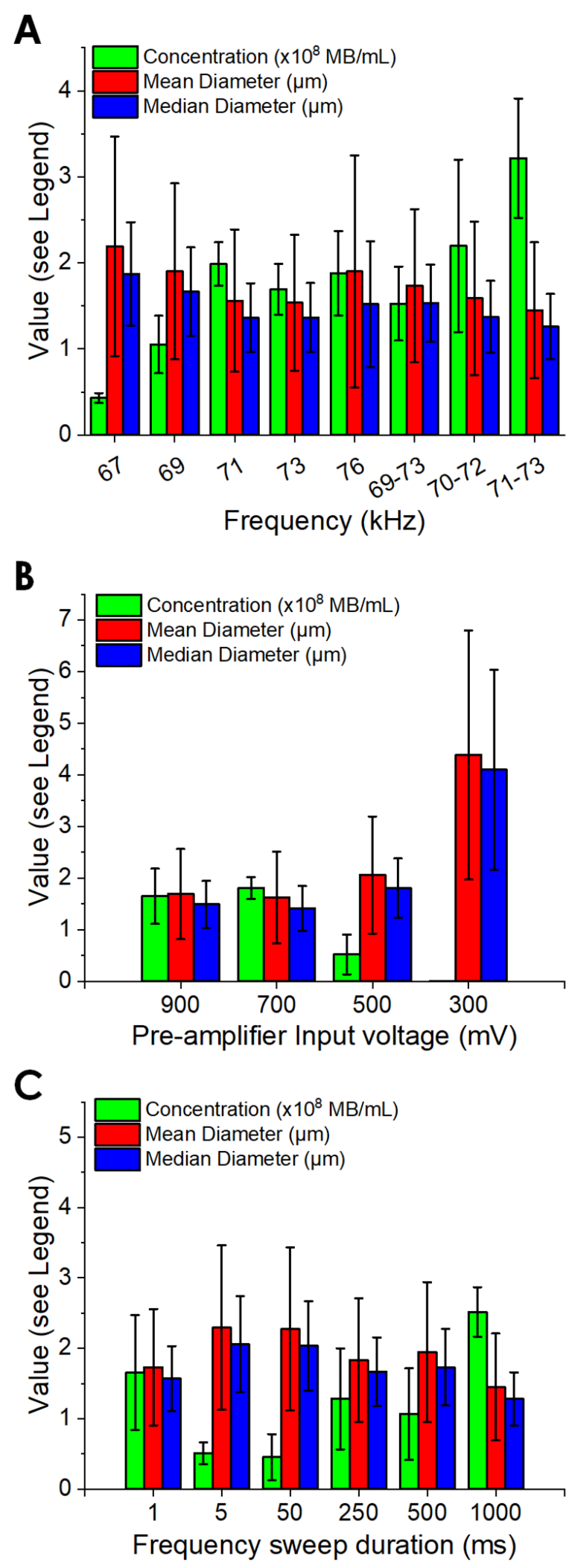
443 FIG. 3. (A) Effect of driving acoustic frequency on microbubble properties. The graph shows the

444 population statistics of microbubbles generated at varying acoustic frequencies, at both single

445 frequency and frequency modulation actuation modes. The piezoelectric transducer was operated at a

446 pre-amplifier input voltage of $900 \mathrm{mV}$. The sweeping frequency groups (69-73, 70-72 and 71-73 kHz)

447 used a linear frequency sweep over $50 \mathrm{~ms}$. The optimum waveform frequency was chosen as a 71-73

$448 \mathrm{kHz}$ frequency sweep. $\mathrm{n}=3$ per frequency. The error bars indicate one standard deviation. (B) Effect

449 of acoustic power on microbubble properties. The graph shows the population statistics of

450 microbubbles generated at varying acoustic pre-amplifier input voltage. The piezoelectric transducer

451 was run using a frequency sweep of $71-73 \mathrm{kHz}$ over $50 \mathrm{~ms}$. The optimum waveform pre-amplifier

452 input voltage was chosen as $900 \mathrm{mV}$, although $700 \mathrm{mV}$ provided microbubbles of comparable

453 characteristics and would also be useable. $n=3$ per input voltage. (C) The graph shows the effect of

454 frequency sweep duration on microbubble properties. Population statistics of microbubbles generated

455 at varying acoustic frequency sweep duration. The piezoelectric transducer was run using a frequency

456 sweep of $71-73 \mathrm{kHz}$ at pre-amplifier input voltage of $900 \mathrm{mV}$. The optimum waveform sweep duration

457 was chosen as $1000 \mathrm{~ms} . \mathrm{n}=3$ per frequency sweep, except $50 \mathrm{~ms}$ which is $\mathrm{n}=2$.

458

459

460

461

462 device units from the custom-built holder. Devices were all operated using a frequency sweep of 71-

$46373 \mathrm{kHz}$ over $1000 \mathrm{~ms}$, at a pre-amplifier input voltage of $900 \mathrm{mV}$. The size and concentration of

464 microbubbles produced with each device replica are reported in Figure 4 (Devices A, B, and C),

465 together with those of the device previously utilised during performance optimisation tests (Device C,

466 Figure $3 \mathrm{C})$. The microbubble concentration varied in the range $1.76-3.77 \times 10^{8}$ microbubbles $/ \mathrm{mL}$, 
467 with an average between devices of $2.65 \pm 0.83 \times 10^{8}$ microbubbles $/ \mathrm{mL}$. The mean microbubble 468 diameter varied in the range $1.45-2.92 \mu \mathrm{m}$, and the average between devices was $2.34 \pm 0.64 \mu \mathrm{m}$.

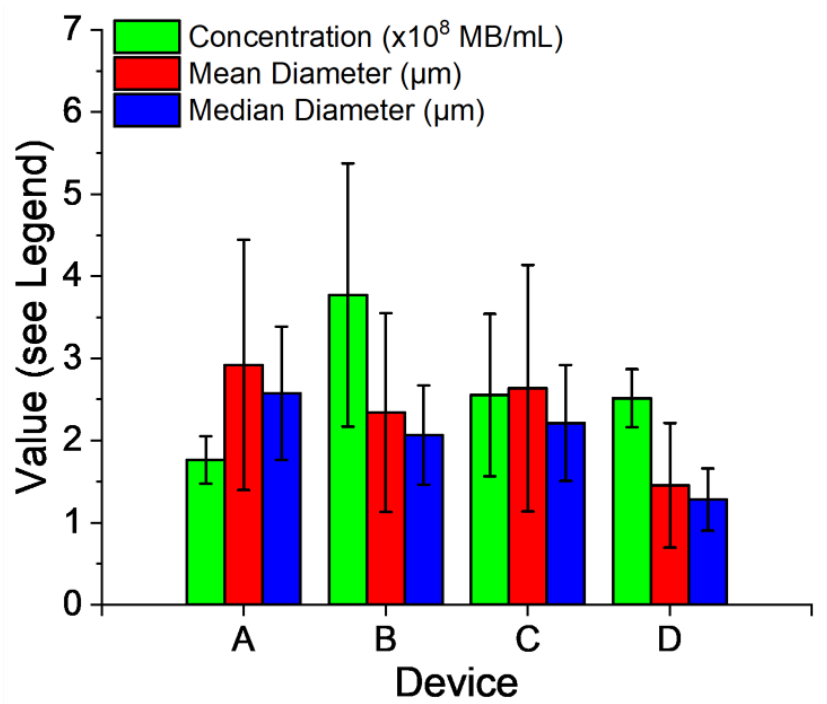

470 FIG. 4. Reproducibility of sonofluidic device performance. The graph shows the population statistics

471 of microbubbles generated at a frequency sweep in the range $71-73 \mathrm{kHz}$ over $1000 \mathrm{~ms}$, at a pre-

472 amplifier input voltage of $900 \mathrm{mV}$. In each group of experiments, a different microfluidic device was

473 employed. The lipid formulation used was a $2 \mathrm{mg} / \mathrm{mL}$ DSPC:PEG40S. $\mathrm{n}=3$ per device.

474 Differences in performance between devices could be potentially attributed to discrepancies in the 475 positioning of the piezoelectric element relative to the microfluidic channel architecture. This could 476 be due to the nature of the manufacturing process, whereby the PDMS layer was manually bonded to 477 the glass substrate. There may have also been differences in the thickness of the glycerol coupling 478 layer between devices, which may have affected the acoustic energy field within the microfluidic 479 channels. Moreover, using a commercial transducer with built-in case, may reduce performance 480 sensitivity to changes in the environmental conditions. Overall, considering the cost-effective and 481 easy-to-perform nature of the manufacturing method employed in this study, all sonofluidic device 482 replicas were capable of producing phospholipid-shelled microbubbles at rates $>10^{6}$ microbubbles $/ \mathrm{s}$ 483 and with a clinically acceptable diameter. 


\section{Comparison with conventional batch sonication}

485 DSPC:PEG40S (9:1) microbubbles were prepared both by batch sonication and using the 486 sonofluidic device, and their size distribution and concentration were monitored over 30 minutes in a

487 hemocytometer. The normalised average microbubble concentration and mean diameter are reported

488 in Figures 5A and 5B, respectively. The microbubble suspensions produced by batch sonication had 489 a larger initial mean diameter $(2.65 \mu \mathrm{m})$ compared to those produced using the sonofluidic device $490(1.75 \mu \mathrm{m})$, and also contained microbubbles with diameter $>10 \mu \mathrm{m}$. The concentration of 491 microbubbles produced by batch sonication was greater than the ones produced using the sonofluidic 492 device, but of the same order of magnitude $\left(4.34 \times 10^{8}\right.$ microbubbles $/ \mathrm{mL} v$ s. $1.71 \times 10^{8}$ 493 microbubbles/mL).
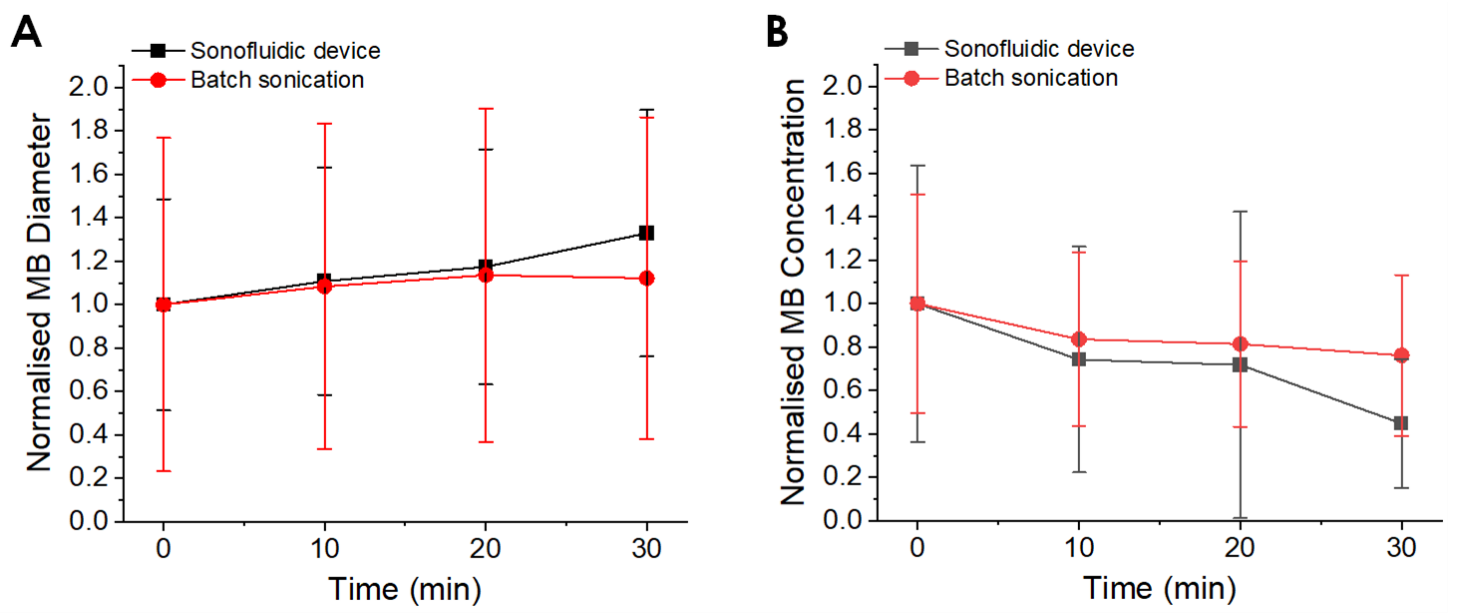

495 FIG. 5. Comparison of the stability of microbubbles produced by batch sonication and the sonofluidic 496 device. Changes in (A) normalised mean diameter and (B) normalised concentration of microbubbles 497 produced by sonication (red circles and line) and the sonofluidic device (black squares and line) over 49830 minutes, measured from bright field microscope images. The sonofluidic device was operated using 499 a frequency sweep in the range $71-73 \mathrm{kHz}$ over $1000 \mathrm{~ms}$, at a pre-amplifier input voltage of $900 \mathrm{mV}$. $500 \mathrm{n}=3$ per production method. 
As shown in Figure 5, both types of microbubble underwent a comparable gradual decrease in

502 concentration and increase in mean diameter over a period of 30 minutes. A two-sample $t$-test was

503 performed on data pairs, and there were no statistically significant differences in microbubble

504 properties between the two production techniques, suggesting that microbubbles produced using the

505 sonofluidic device have comparable stability to those produced using conventional batch sonication.

\section{Modification of microbubble formulation}

507 The total lipid concentration of DSPC:PEG40S was varied in the range $2-6 \mathrm{mg} / \mathrm{mL}$, to determine

508 whether the amount of phospholipid had an effect on the characteristics of microbubbles produced 509 using the sonofluidic device (see Figure 6). It was found that increasing the lipid concentration from 5102 to $6 \mathrm{mg} / \mathrm{mL}$ increased microbubble concentration from $0.45 \pm 0.17 \times 10^{8}$ microbubbles $/ \mathrm{mL}$ to

$5112.12 \pm 0.45 \times 10^{8}$ microbubbles $/ \mathrm{mL}$, likely due to the greater number density of phospholipid

512 molecules readily available to stabilise the gas-liquid interface of the forming microbubbles. The

513 increase in microbubble concentration was however less pronounced when the lipid concentration

514 was varied from 4 to $6 \mathrm{mg} / \mathrm{mL}\left(1.78 \pm 0.42 \times 10^{8}\right.$ microbubbles $/ \mathrm{mL}$ vs. $2.12 \pm 0.45 \times 10^{8}$

515 microbubbles $/ \mathrm{mL}$ ). The mean microbubble diameter did not show significant changes as a function

516 of the total lipid concentration, and was in the range 1.67-1.99 $\mu \mathrm{m}$. An alternative formulation, similar

517 to that used in the commercial agent Definity ${ }^{\circledR}$, was also tested using a $2 \mathrm{mg} / \mathrm{mL}$ total lipid

518 concentration. microbubble shell constituents in this formulation were DPPC, DSPE-mPEG5000,

519 and DPPA (at a molar ratio of 8:1:1). Unlike the DSPC:PEG40S microbubbles, the resuspension

520 solvent was a more viscous solution of water, glycerol, and propylene glycol. The average microbubble

521 concentration with this formulation was significantly greater than for DSPC:PEG40S, and equal to

$5227.79 \pm 2.43 \times 10^{8}$ microbubbles $/ \mathrm{mL}$ (corresponding to an average production rate of $6.5 \times 10^{6}$

523 microbubbles/s). The mean microbubble diameter was slightly reduced when compared to the

524 DSPC:PEG40s formulation with the same total lipid concentration $(1.52 \mu \mathrm{m} v$ s. $1.99 \mu \mathrm{m})$. These 
525 observations are consistent with previous studies that reported an increase in microbubble 526 concentration for DPPC-based formulations containing both glycerol and propylene glycol, and 527 produced by mechanical agitation (Daeichin, et al. 2016). This is likely due to the reduced diffusivity 528 of gas in the suspending medium as compared to saline, inhibiting microbubble destruction during 529 processing; propylene glycol is also an effective de-foaming agent and this may help to promote 530 formation of microbubbles over foam. A previous study by Parhizkar et al. also reported on an inverse 531 relationship between diameter and medium viscosity, for microbubbles produced in a capillary 532 embedded T-junction device (Parhizkar, et al. 2015). Moreover, the greater viscosity of the suspension 533 medium in the Definity®-like formulation likely increased the overall microbubble lifetime.

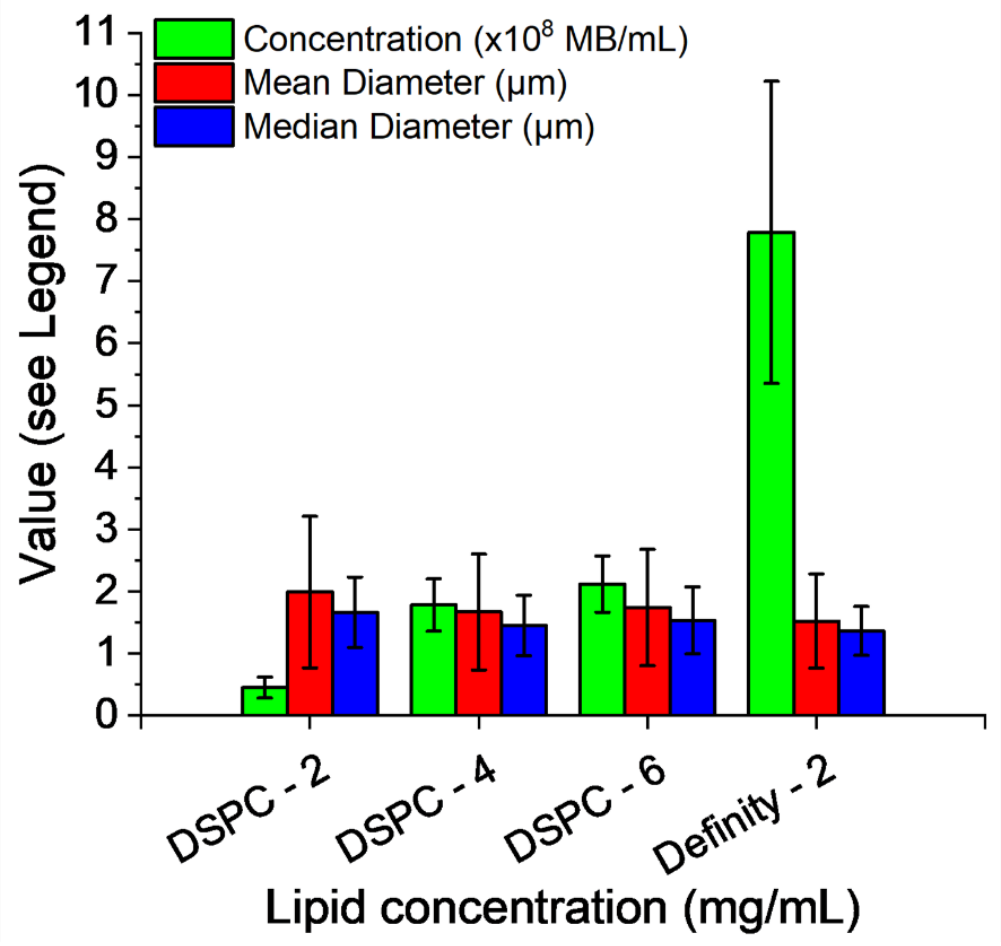

535 FIG. 6. Effect of total lipid concentration and formulation on the characteristics of microbubbles

536 produced using the sonofluidic device. The graph shows the population statistics of microbubbles 537 generated at a frequency sweep in the range $71-73 \mathrm{kHz}$ over $1000 \mathrm{~ms}$, at a pre-amplifier input voltage 538 of $900 \mathrm{mV}$ and using different lipid concentrations of DSPC:PEG40S and a Definity®-like 
539 formulation. Optimisation of lipid concentration and formulation can improve microbubble

540 production rates. $n=3$ per formulation.

\section{E. Demonstration of scaled-up microbubble production}

542 Microbubbles were produced using the sonofluidic device described in section II.D, which

543 consisted of a scaled-up microfluidic channel architecture operated at greater volumetric flow rates

544 and driving acoustic power. The lipid suspension in these experiments comprised DSPC and PEG40S

545 at a molar ratio of 9:1, suspended in a water, glycerol and propylene glycol mixture (80:10:10 v/v

546 respectively). Notably, the microbubble production rate for this device directly correlated with the

547 inlet flow rate, and increased from $0.18 \times 10^{8}$ microbubbles $/ \mathrm{s}$ (at $5 \mathrm{~mL} / \mathrm{min}$ ) up to a maximum of

$5481.18 \times 10^{8}$ microbubbles $/ \mathrm{s}$ (at $35 \mathrm{~mL} / \mathrm{min}$ ) for a single device, as shown in Figure $7 \mathrm{~A}$.
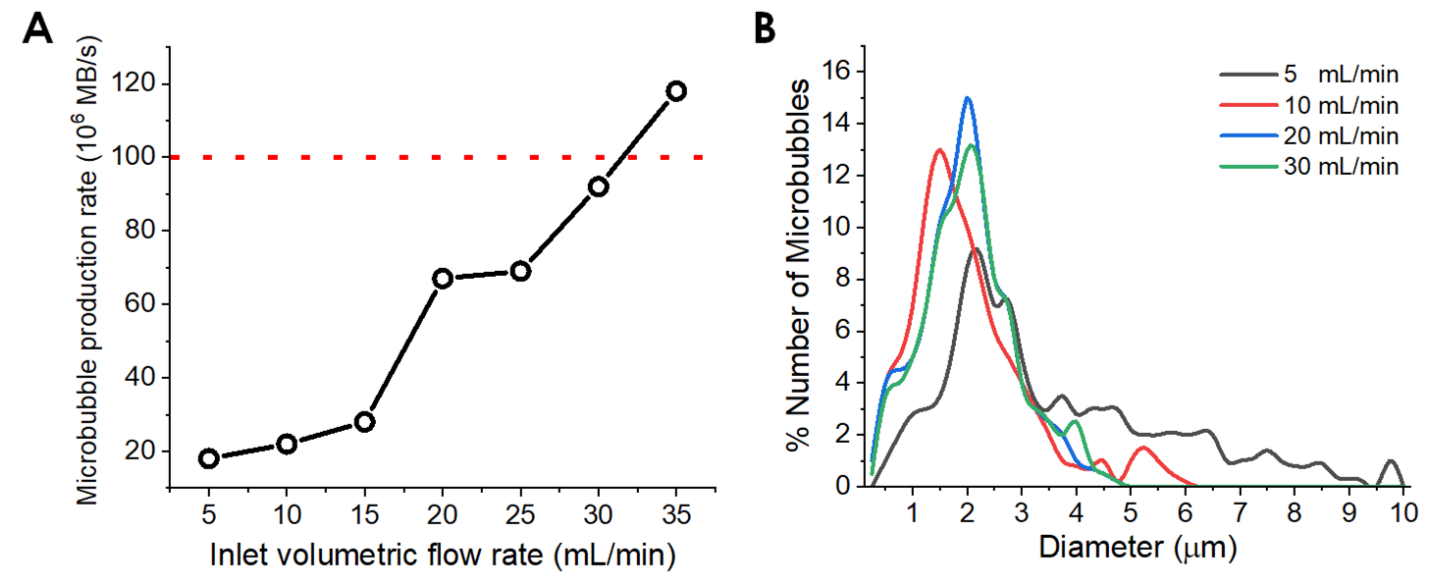

550 FIG. 7. (A) Microbubble production rate as a function of the inlet volumetric flow rate of the

551 phospholipid suspension, obtained using the scaled-up sonofluidic device (driven using a $30 \mathrm{~W}$

552 transducer). The dotted red horizontal line corresponds to a production rate of 100 millions of

553 microbubbles per second. (B) microbubbles size distribution at four different inlet volumetric flow

554 rates. The lipid suspension in these experiments comprised DSPC and PEG40S at a molar ratio of

555 9:1, and the suspension medium was a mixture of water, glycerol and propylene glycol (80:10:10 v/v). 
557 configuration described above and other methods that have been published (Table 1). Volumetric 558 flow rates $>35 \mathrm{~mL} / \mathrm{min}$ resulted in delamination of the PDMS from the glass substrate, and 559 subsequent leakage. However, greater production rates may be achievable by improving the PDMS-

560 glass bonding strength through optimisation of the plasma treatment process. As shown in Figure 7B, 561 the microbubble size dispersity reduced with increasing the volumetric flow rate from $5 \mathrm{~mL} / \mathrm{min}$ to $56220 \mathrm{~mL} / \mathrm{min}$; however, it remained substantially unchanged at flow rates $\geq 20 \mathrm{~mL} / \mathrm{min}$. The greater 563 size dispersity at the lower flow rates requires further investigations, but may be due to the increased 564 transit time of precursor bubbles across the ultrasound field that may have potentially resulted in 565 enhanced microbubble fragmentation and coalescence.

\section{F. Limitations and future development for microbubble production}

567 The results from this study demonstrate the feasibility of the proposed sonofluidic method as a 568 means of producing microbubbles with a clinically relevant composition and size distribution, and in

569 a continuous-flow format. The mechanism of microbubble formation was not explicitly investigated.

570 It is hypothesised that standing surface waves were established at the gas-liquid interface of the larger

571 precursor bubbles, and that the resulting surface oscillations led to the entrapment and 'release' of

572 smaller microbubbles, consistent with the observations reported by Ohl et al. (Ohl, et al. 2010). Further

573 work is required to test this hypothesis using high speed imaging as the device architecture and

574 microbubble formulation are different.

575 To the best of the authors' knowledge, this is the first study reporting on the use of this approach

576 to produce microbubbles stabilised with clinically relevant formulations of coating material, including

577 a mimic of the clinically approved contrast agent Definity ${ }^{\circledR}$. Compared to conventional batch

578 sonication or other two-stage methods relying on ultrasound exposure of precursor bubbles (Chen, et

579 al. 2014), the microbubbles produced using the sonofluidic device could be directly administered 
580 intravenously without the need for post-production fractionation or centrifugation processes that are

581 typically required to eliminate microbubbles with diameter $>10 \mu \mathrm{m}$. It will be necessary, however, to

582 confirm that the coating properties and acoustic response of the microbubbles are also comparable to

583 those of microbubbles produced via sonication.

584 The proposed sonofluidic strategy can produce microbubbles at rates of $10^{8}$ per second using a 585 single channel, which is significantly greater than that achievable with conventional microfluidic 586 approaches and comparable to batch sonication. Production rates of $>10^{9}$ microbubbles per second 587 could easily be achieved through parallel actuation of multiple channels within a single platform; and 588 even higher rates using multiple devices. A further important point is that the risk of sample 589 contamination from erosion of the sonicator tip is also removed, as there is no direct contact between 590 the microbubble suspension and the ultrasound transducer as in batch sonication. This may be 591 advantageous for good manufacturing practice (GMP) compliance, and for producing microbubble 592 formulations loaded with bioactive compounds. The risk of clogging associated with conventional 593 microfluidic devices is also minimized and encapsulation efficiency expected to be significantly higher 594 than for conventional emulsification. This will likely be beneficial for the preparation of multi595 component bubbles, e.g. surface functionalised with targeting ligands or containing solid particles, for 596 which clogging is a greater risk. It would also be comparatively simple to add a secondary channel to 597 the device to facilitate subsequent reaction with a functional component. e.g. microbubbles could be 598 generated with a biotinylated lipid and then functionalised by exposing them to an avidin 599 functionalised drug molecule or targeting ligand. Production of functionalized microbubbles has yet 600 to be demonstrated, however, and this will be evaluated in future investigations, together with a 601 broader range of clinically applicable microbubble shell constituents.

602 There are other aspects of the developed system that also require improvement. These include (i) 603 the repeatability of the microfluidic device manufacturing process, and particularly of the relative 
604 positioning of the PDMS and glass layers, (ii) the repeatability of the coupling process between the

605 piezoelectric element and glass carrier, and (iii) the use of scalable and high volume capacity fluid 606 supply units (e.g., pressurised reservoirs) as an alternative to syringe pumps. The results of this study

607 also suggest that varying the input acoustic intensity may provide an effective means of controlling

608 the microbubble size, although this may also result in a change in microbubble concentration. Future 609 studies will investigate whether operating the device over a broader range of ultrasound frequencies

610 (i.e., including harmonics of the transducer fundamental resonance frequency) would offer a method

611 for tuning microbubble size and achieving a narrower size distribution.

\section{IV. CONCLUSIONS}

614 The feasibility of producing microbubbles with clinically relevant size $(1-2 \mu \mathrm{m})$ and

615 composition using a sonofluidic device was demonstrated. The microbubble diameter, concentration 616 and stability were comparable with those achieved with batch sonication, but with a narrower size

617 distribution and importantly no microbubbles larger than $<5 \mu \mathrm{m}$ in diameter. This removes the need

618 for post-production fractionation. Production rates of $>10^{8}$ microbubbles per second were achieved 619 using a single device. These are comparable with production rates associated with batch sonication, 620 but the risk of contamination and/or degradation of sensitive components is removed. The device

621 can also be operated continuously, reducing the risk of batch to batch variation. Further work is

622 needed to elucidate the mechanism of microbubble formation within the device and to characterize

623 the microbubble surface and acoustic properties.

\section{$624 \quad$ ACKNOWLEDGEMENTS}

625 This work was supported by the Institute of Engineering and Technology (AF Harvey Prize)

626 and the Engineering and Physical Sciences Research Council (grants EP/I021795/1 and 
EP/L025825/1). The authors gratefully acknowledge the help of Mr James Fisk in the University of

628 Oxford workshop for assistance in device manufacturing.

629

630

631

632

633

634

635

636

637

638

639

640

641

642

643

644

645

646

647

648

649

650

651

652

653

654

655

656

657

658

659

660

661

662

663

664

665

666

667

668

669

670

\section{REFERENCES}

Al-Jawadi S, Thakur SS. Ultrasound-responsive lipid microbubbles for drug delivery: A review of preparation techniques to optimise formulation size, stability and drug loading. International journal of pharmaceutics 2020:119559.

Alter J, Sennoga CA, Lopes D, Eckersley RJ, Wells DJ. Microbubble stability is a major determinant of the efficiency of ultrasound and microbubble mediated in vivo gene transfer. Ultrasound in medicine \& biology 2009; 35:976-84.

Ankrett DN, Carugo D, Lei J, Glynne-Jones P, Townsend PA, Zhang X, Hill M. The effect of ultrasoundrelated stimuli on cell viability in microfluidic channels. Journal of nanobiotechnology 2013; 11:1-5.

Borden MA, Kruse DE, Caskey CF, Zhao S, Dayton PA, Ferrara KW. Influence of lipid shell physicochemical properties on ultrasound-induced microbubble destruction. IEEE transactions on ultrasonics, ferroelectrics, and frequency control 2005; 52:1992-2002.

Browning RJ, Aron M, Booth A, Rademeyer P, Wing S, Brans V, Shrivastava S, Carugo D, Stride E. Spectral Imaging for Microbubble Characterization. Langmuir 2019; 36:609-17.

Carugo D, Lee JY, Pora A, Browning RJ, Capretto L, Nastruzzi C, Stride E. Facile and cost-effective production of microscale PDMS architectures using a combined micromilling-replica moulding ( $\mu \mathrm{Mi}-\mathrm{REM}$ ) technique. Biomedical microdevices 2016; 18:4.

Carugo D, Octon T, Messaoudi W, Fisher AL, Carboni M, Harris NR, Hill M, Glynne-Jones P. A thinreflector microfluidic resonator for continuous-flow concentration of microorganisms: a new approach to water quality analysis using acoustofluidics. Lab on a Chip 2014; 14:3830-42.

Carugo D, Owen J, Crake C, Lee JY, Stride E. Biologically and acoustically compatible chamber for studying ultrasound-mediated delivery of therapeutic compounds. Ultrasound in medicine \& biology 2015; 41:1927-37.

Castro-Hernández E, Van Hoeve W, Lohse D, Gordillo JM. Microbubble generation in a co-flow device operated in a new regime. Lab on a Chip 2011; 11:2023-29.

Chen H, Li J, Zhou W, Pelan EG, Stoyanov SD, Arnaudov LN, Stone HA. Sonication-Microfluidics for Fabrication of Nanoparticle-Stabilized Microbubbles. Langmuir 2014; 30:4262-66.

Daeichin V, van Rooij T, Skachkov I, Ergin B, Specht PA, Lima A, Ince C, Bosch JG, van der Steen AF, de Jong $\mathrm{N}$. Microbubble composition and preparation for high-frequency contrast-enhanced ultrasound imaging: in vitro and in vivo evaluation. IEEE transactions on ultrasonics, ferroelectrics, and frequency control 2016; 64:555-67.

Dewitte H, Roovers S, De Smedt SC, Lentacker I. Enhancing nucleic acid delivery with ultrasound and microbubbles. Nanotechnology for Nucleic Acid Delivery: Springer, 2019. 241-51.

Dhanaliwala AH, Chen JL, Wang S, Hossack JA. Liquid flooded flow-focusing microfluidic device for in situ generation of monodisperse microbubbles. Microfluidics and nanofluidics 2013; 14:457-67.

Dollet B, van Hoeve W, Raven J-P, Marmottant P, Versluis M. Role of the channel geometry on the bubble pinch-off in flow-focusing devices. Physical review letters 2008; 100:034504.

Ferrara K, Pollard R, Borden M. Ultrasound microbubble contrast agents: fundamentals and application to gene and drug delivery. Annu. Rev. Biomed. Eng. 2007; 9:415-47.

Feshitan JA, Chen CC, Kwan JJ, Borden MA. Microbubble size isolation by differential centrifugation. Journal of colloid and interface science 2009; 329:316-24. 
671

672

673

674

675

676

677

678

679

680

681

682

683

684

685

686

687

688

689

690

691

692

693

694

695

696

697

698

699

700

701

702

703

704

705

706

707

708

709

710

711

712

713

714

715

716

Frinking $P$, Segers T, Luan Y, Tranquart F. Three decades of ultrasound contrast agents: a review of the past, present and future improvements. Ultrasound in medicine \& biology 2020; 46:892-908.

Garg S, Thomas AA, Borden MA. The effect of lipid monolayer in-plane rigidity on in vivo microbubble circulation persistence. Biomaterials 2013; 34:6862-70.

Garstecki P, Fuerstman MJ, Stone HA, Whitesides GM. Formation of droplets and bubbles in a microfluidic T-junction-scaling and mechanism of break-up. Lab on a Chip 2006; 6:437-46.

Gnyawali V, Moon B-U, Kieda J, Karshafian R, Kolios MC, Tsai SS. Honey, I shrunk the bubbles: microfluidic vacuum shrinkage of lipid-stabilized microbubbles. Soft Matter 2017; 13:4011-16.

Hettiarachchi K, Talu E, Longo ML, Dayton PA, Lee AP. On-chip generation of microbubbles as a practical technology for manufacturing contrast agents for ultrasonic imaging. Lab on a Chip 2007; 7:46368.

Hosny NA, Mohamedi G, Rademeyer P, Owen J, Wu Y, Tang M-X, Eckersley RJ, Stride E, Kuimova MK. Mapping microbubble viscosity using fluorescence lifetime imaging of molecular rotors. Proceedings of the National Academy of Sciences 2013; 110:9225-30.

Jiang $X$, Zhang $Y$, Edirisinghe $M$, Parhizkar M. Combining microfluidic devices with coarse capillaries to reduce the size of monodisperse microbubbles. RSC advances 2016; 6:63568-77.

Jonnalagadda US, Hill M, Messaoudi W, Cook RB, Oreffo RO, Glynne-Jones P, Tare RS. Acoustically modulated biomechanical stimulation for human cartilage tissue engineering. Lab on a Chip 2018; 18:473-85.

Kooiman K, Vos HJ, Versluis M, de Jong N. Acoustic behavior of microbubbles and implications for drug delivery. Advanced drug delivery reviews 2014; 72:28-48.

Leibacher I, Schatzer S, Dual J. Impedance matched channel walls in acoustofluidic systems. Lab on a Chip 2014; 14:463-70.

Manneberg O, Vanherberghen B, Önfelt B, Wiklund M. Flow-free transport of cells in microchannels by frequency-modulated ultrasound. Lab on a Chip 2009; 9:833-37.

OhI S-W, Ow DS-W, Klaseboer E, Wong VV, Camattari A, Ohl C-D. Creation of cavitation activity in a microfluidic device through acoustically driven capillary waves. Lab on a Chip 2010; 10:1848-55.

Owen J, Kamila S, Shrivastava S, Carugo D, Bernardino de la Serna J, Mannaris C, Pereno V, Browning R, Beguin E, McHale AP. The Role of PEG-40-stearate in the Production, Morphology, and Stability of Microbubbles. Langmuir 2018; 35:10014-24.

Pahlavan AA, Stone HA, McKinley GH, Juanes R. Restoring universality to the pinch-off of a bubble. Proceedings of the National Academy of Sciences 2019; 116:13780-84.

Parhizkar M, Edirisinghe M, Stride E. Effect of operating conditions and liquid physical properties on the size of monodisperse microbubbles produced in a capillary embedded T-junction device. Microfluidics and nanofluidics 2013; 14:797-808.

Parhizkar M, Edirisinghe M, Stride E. The effect of surfactant type and concentration on the size and stability of microbubbles produced in a capillary embedded T-junction device. Rsc Advances 2015; 5:10751-62.

Parhizkar M, Stride E, Edirisinghe M. Preparation of monodisperse microbubbles using an integrated embedded capillary T-junction with electrohydrodynamic focusing. Lab on a Chip 2014; 14:243746.

Peyman SA, Abou-Saleh RH, McLaughlan JR, Ingram N, Johnson BR, Critchley K, Freear S, Evans JA, Markham AF, Coletta PL. Expanding 3D geometry for enhanced on-chip microbubble production and single step formation of liposome modified microbubbles. Lab on a Chip 2012; 12:4544-52.

Rademeyer P, Carugo D, Lee JY, Stride E. Microfluidic system for high throughput characterisation of echogenic particles. Lab on a Chip 2015; 15:417-28. 
Rickel JR, Dixon AJ, Klibanov AL, Hossack JA. A flow focusing microfluidic device with an integrated Coulter particle counter for production, counting and size characterization of monodisperse microbubbles. Lab on a Chip 2018; 18:2653-64. ultrasound contrast agent synthesis by flow-focusing through multi-gas-component microbubble stabilization. Applied Physics Letters 2020; 116:173701.

Segers T, Lohse D, Versluis M, Frinking P. Universal equations for the coalescence probability and longterm size stability of phospholipid-coated monodisperse microbubbles formed by flow focusing. Langmuir 2017; 33:10329-39.

Sennoga CA, Yeh JS, Alter J, Stride E, Nihoyannopoulos P, Seddon JM, Haskard DO, Hajnal JV, Tang M-X, Eckersley RJ. Evaluation of methods for sizing and counting of ultrasound contrast agents. Ultrasound in medicine \& biology 2012; 38:834-45.

Seo M, Gorelikov I, Williams R, Matsuura N. Microfluidic Assembly of Monodisperse, NanoparticleIncorporated Perfluorocarbon Microbubbles for Medical Imaging and Therapy. Langmuir 2010; 26:13855-60.

Sirsi S, Feshitan J, Kwan J, Homma S, Borden M. Effect of microbubble size on fundamental mode high frequency ultrasound imaging in mice. Ultrasound in medicine \& biology 2010; 36:935-48.

Stride E, Edirisinghe M. Novel microbubble preparation technologies. Soft matter 2008; 4:2350-59.

Stride E, Saffari N. Microbubble ultrasound contrast agents: a review. Proceedings of the Institution of Mechanical Engineers, Part H: Journal of Engineering in Medicine 2003; 217:429-47.

Stride E, Segers T, Lajoinie G, Cherkaoui S, Bettinger T, Versluis M, Borden M. Microbubble agents: New directions. Ultrasound in medicine \& biology 2020; 46:1326-43.

van Elburg B, Collado-Lara G, Bruggert GW, Segers T, Versluis M, Lajoinie G. Feedback-controlled microbubble generator producing one million monodisperse bubbles per second. Rev Sci Instrum 2021; 92. 